\title{
Modeling Enhanced Adsorption of Explosive Molecules on a Hydroxylated Graphene Pore
}

\author{
Ronald Scott Holt, Thomas R. Rybolt (i) \\ Department of Chemistry and Physics, University of Tennessee at Chattanooga, Chattanooga, TN, USA \\ Email: Tom-Rybolt@utc.edu
}

How to cite this paper: Holt, R.S. and Rybolt, T.R. (2019) Modeling Enhanced Adsorption of Explosive Molecules on a Hydroxylated Graphene Pore. Graphene, 8, 1-18.

https://doi.org/10.4236/graphene.2019.81001

Received: January 12, 2019

Accepted: January 28, 2019

Published: January 31, 2019

Copyright $\odot 2019$ by author(s) and Scientific Research Publishing Inc. This work is licensed under the Creative Commons Attribution International License (CC BY 4.0).

http://creativecommons.org/licenses/by/4.0/

\begin{abstract}
The possibility of a graphene bilayer nanosensor for the detection of explosive molecules was modeled using computational chemistry. A pore was designed on a graphene bilayer structure with three strategically placed perimeter hydroxyl $(\mathrm{OH})$ groups built around the edge of an indented, two-dimensional hexagonal pore. This hydroxylated pore and models of various explosive molecules were optimized using MM2 molecular mechanics parameters. Values were calculated for the molecule-surface interaction energy (binding energy), E, for 22 explosive molecules on a flat graphene bilayer and on the specially designed hydroxylated pore within the bilayer. The molecule-surface binding energy for trinitrotoluene (TNT) increased from $17.9 \mathrm{kcal} / \mathrm{mol}$ on the flat graphene bilayer to $42.3 \mathrm{kcal} / \mathrm{mol}$ on the hydroxylated pore. Due to the common functionality of nitro groups that exist on many explosive molecules, the other explosive molecules studied gave similar enhancements based on the specific hydrogen bonding interactions formed within the pore. Each of the 22 explosive adsorbate molecules showed increased molecule-surface interaction on the bilayer hydroxylated pore as compared to the flat bilayer. For the 22 molecules, the average $\mathrm{E}$ for the flat graphite surface was 15.8 $\mathrm{kcal} / \mathrm{mol}$ and for the hydroxylated pore $\mathrm{E}$ was $33.8 \mathrm{kcal} / \mathrm{mol}$. An enhancement of adsorption should make a detection device more sensitive. Nanosensors based on a modified graphene surface may be useful for detecting extremely low concentrations of explosive molecules or explosive signature molecules.
\end{abstract}

\section{Keywords}

Molecular Mechanics, Explosives, Molecule-Surface Binding Energy, Hydroxylated Graphene Pore, Graphene Bilayer, Enhanced Adsorption for Explosives 


\section{Introduction}

The unique and unusual structural and electrical properties of graphene have led to the development of graphene-based chemical sensors and biosensors of varied structures and fabrication methods. For example, resistivity changes on graphene-based sensors have been used for gas phase sensing at low levels. Graphene-based electronic devices that can act as sensors for specific molecules; or for environmental properties such as temperature, light, and moisture; or more generally as electronic noses have been of interest in recent years [1]. Graphene nanostructures have been integrated into field-effect transistor (FET) and chemiresistive devices to obtain sensing properties [2].

One of the most promising applications of graphene is its use as achemical or gas sensor although challenges remain [3]. Surface functionalized graphenes have been found to be extremely useful [4]. One example of the advantage of surface modification has been the use of an ozone treatment to modify a graphene surface. This modification resulted in going from a pristine graphene sensor with a detection limit above $10 \mathrm{ppm}$ for $\mathrm{NO}_{2}$ to a sensor with a limit of $1.3 \mathrm{ppb}[5]$.

There has been a continuing desire for the development of devices capable of detecting explosive molecules in trace amounts [6]. This need is related to the use of homemade explosive devices used in personal, package, and roadside bombings [6] [7]. There are existing techniques for preemptive detection of explosive molecules including ion mobility spectrometry and mass spectrometry. However, the desire for new techniques and devices has continued and portable and hand-held device have been explored. Experiments have been done using microcantilevers [8]. Graphene-based sensors capable of detecting low concentrations of specific hazardous gases or vapor phase explosive molecules are of great interest [9]. A device that could detect trace amounts of explosive molecules from a distance, as well as easy portability, would be particularly useful [8]. Toward that end, our current study relates to modeling the ability of a graphene nanosensor pore to be designed to enhance the attraction of common explosive molecules [10] [11].

A variety of graphene-based chemical sensors have been developed, fabricated, and tested [12]-[19]. Results from these devices demonstrate that graphene is a material suitable for constructing multifunctional sensors for varied applications [12]. Graphene sensors have been fabricated by different means including conventional photolithography [13]. Graphene's electrical properties are sensitive to temperature, light, and humidity along with other environmental signals such as gas phase molecules. They may be used for multifunctional sensors if a technique to decouple various types of environment signals is used [14]. The combination of principle component analysis (PCA) and prediction accuracies demonstrated the capability of an unmodified graphene chemical vapor sensor [15].

Graphene sensors have been used for monitoring $\mathrm{NO}_{2}$ concentrations in the 
range of $10-154 \mathrm{ppb}$ [16]. The sensitivity to $\mathrm{NO}_{2}$ increased significantly when the sensor was operated at $150^{\circ}$ where cross-selectivity to water, $\mathrm{SO}_{2}$, and other gases was minimized [16]. One approach has been to include multiple surfaces in an array. For example, a 32-element chemiresistive sensor array with nine different sensor materials was constructed and was able to operate in the $1-30$ ppm concentration range [17]. The value of surface modified sensors has been demonstrated with graphene and poly (methyl methacrylate) composite laminates that have been prepared using chemical vapor deposition (CVD) for volatile organic compound detection [18]. Sensitivity has been increased by introducing defects into a surface and a sub-ppb level detection limit was created [12]. An alternate approach was to hydrogenate a graphene surface. This hydrogenated graphene sensor proved to be a stable $\mathrm{NO}_{2}$ sensor at room temp [19].

For detection, signal properties from a graphene sensor along with the surface interactions and adsorption of explosive molecules on the surface must be considered. A device containing a graphene sensor or sensors would have to be able to attract vapor samples in the air, transduce adsorbed molecules as a signal, and be able to effectively characterize the data [8]. An ideal graphene sensor would be characterized by an affinity for preferentially attracting explosive molecules.

Computational studies have been carried out to gain more information about possible molecule-surface interactions. Prior density functional theory (DFT) calculations have explored molecule-graphene and molecule-modified graphene surface interactions [20] [21] [22] [23]. The binding energy of $\mathrm{H}_{2} \mathrm{O}, \mathrm{NH}_{3}, \mathrm{CO}$, $\mathrm{NO}_{2}$, and $\mathrm{NO}$ on a graphene substrate was investigated [20]. The results of a DFT study found that $\mathrm{Si}$-doped graphene could be a promising nanosensor for cyanogen chloride $(\mathrm{ClCN})$ detection [22]. DFT was used to calculate the adsorption of $\mathrm{CO}_{2}$ gas on graphene with several different doped surfaces and the graphene doped with $\mathrm{Al}$ was a possibility for a sensitive gas sensor [23]. Surface hydroxyl groups can promote the adsorption of $\mathrm{NH}_{3}$ on the graphene oxide (GO), and the enhancement of the binding energy with the hydroxyl groups was found [21].

Adsorption studies involving the surface interactions of graphene with organic molecules using force field calculations indicate significant noncovalent interactions can exist between a graphene surface and a molecule [24]. These forces are primarily due to van der Waals (vdW) attractions and have been experimentally demonstrated and effectively modeled [24]. Organic explosive molecules exhibit similarities in structure and functionality, that may lead to a common two-dimensional pore capable of significantly enhancing adsorption. In prior work force field calculations using molecular mechanics have been shown to compare well with experimental binding energies. However, only those DFT calculations that included a dispersion correction were observed to provide accurate binding energies [24].

An explosophore is the part of a molecule or functional group that makes a compound explosive. Nitrogen and oxygen containing explosophores are particularly strong because, in addition to providing oxygen, they react to form mole- 
cular nitrogen. Nitrogen a very stable molecule, and thus the overall reaction is strongly exothermic [25]. The gas formed expands causing a shock wave. The $\mathrm{NO}_{2}$ nitro group is a common explosophore among a number of common explosive molecules [10] [11] [26]. Although mobile and hand-held detectors have been developed, interest in new methods of detection continues [26].

In this study, the structure of a series of organic molecules used as explosives is modeled with molecular mechanics software (Scigress, Fujitsu). Then the molecule-surface adsorption interaction energies with a bilayer graphene surface are determined along with the interactions for a designed bilayer pore that enhances adsorption. The purpose of this work is to evaluate the adsorption properties of explosive molecules with a conceptual sensor surface pore.

\section{Theory}

Molecule-surface binding energies can be modeled by placing a molecule of interest on a surface site to create an adsorbed state and determining the steric energy of this molecule-surface system, $E_{m s}$. The isolated molecule steric energy, $E_{m}$, and the isolated surface steric energy, $E_{s}$, may also be determined. Here $E$ is the energy required to remove the molecule from the surface site and is given by

$$
E=\left(E_{m}+E_{s}\right)-E_{m s}
$$

Molecular mechanics MM2 force field calculations (Scigress, Fujitsu) were utilized for these energy calculations. As written above, the process represents one of desorption and $\mathrm{E}$ is the energy required to remove the molecule from the surface. Conversely, the adsorption energy would be equal to the negative value of E. A positive desorption energy indicates favorable binding energy of the molecule in its attraction to the surface. For convenience, the binding energies in this work are reported as positive desorption values.

In prior calculations with a graphite model based on multiple layers of graphene, $90 \%$ or more of the van der Waals (vdW) interaction was due to the first layer, $9 \%$ or less due to the second layer and only $1 \%$ or less was due to the third layer [27]. Molecular energy calculated from molecular mechanicsis a sum of the various covalent and noncovalent energies. In this work, van der Waals forces and hydrogen bonding were the dominate forces responsible for molecular adsorption.

In prior work, MM2 parameters used to calculate molecule-surface binding energies compared well to experimental values obtained from gas-solid chromatography (GSC) for isolated molecule physical adsorption [24]. In addition, by considering molecule-molecule nearest neighbor interactions, calculated monolayer coverage binding energies also compared well to thermal program desorption (TPD) experiments. For example, TPD experiments and MM2 calculations for monolayer desorption gave 0.50 and $0.52,0.72$ and 0.71 , and 1.41 and 1.47 $\mathrm{eV}$ for benzene, o-dichlorobenzene, and coronene, respectively [28].

Molecular mechanics provides a simple way to estimate molecule-surface interactions or binding energies without regard to electronic details. While ap- 
proximate, these calculations have compared well to experimental binding energies for a number of molecule-carbon surface systems dominated by van der Waals forces. In this work, vdW and hydrogen bonding are the primary binding forces and the hydrogen bond values are approximated by MM2 force field calculations. In prior comparisons, molecule-surface interactions have not been well approximated by DFT unless dispersion corrections were included [29].

In the Henry's Law region of low coverage, the extent of adsorption for a molecule is largely based on the molecule-surface interaction energy. There is preferential adsorption of the very low concentration vapor of an explosive molecule as its value of $\mathrm{E}$ is increased Because hydrogen bonding plays a central role in the site enhanced explosive adsorption, the humidity of air could play a significant role in this process. For our initial work, our focus is on how to enhance the value of $\mathrm{E}$ and we treat in vacuo. We do not model the adsorption of a multi-component system or deal with the complications of water vapor or other aspects of air.

When calculating the theoretical moles of a low concentration adsorbate adhering to a surface or surface site, the viral coefficient of physical adsorption may be considered [30]. In the Henry's law limit of low coverage, the moles of gas absorbed, $n_{a d s}$ can be directly related to the gas-solid viral coefficient, $B_{2 s}$ as $n_{\text {ads }}=P\left(B_{2 s} / R T\right)$ where $P$ is the partial pressure of the adsorbate, $T$ is the temperature, and $\mathrm{R}$ is the gas constant. In prior work, the moles of gas adsorbed has been approximated on a flat surface model where $A$ is the adsorbent surface area in the xy plane and $\mathrm{z}$ is the axis perpendicular to the surface [30]. Assuming $z^{*}$ is the adsorbate-surface equilibrium separation and the molecule-surface interaction potential, $u$, is represented by a Lennard-Jones $(m, n)$ potential, then an approximation of the exact integral expression given by

$$
B_{2 s}=A z * \sqrt{(2 \pi / m n)} \sqrt{(R T / E)} \exp (E / R T)
$$

where $E$ is the molecule-surface binding energy of and exp is the exponential [30].

\section{Analysis}

Molecular mechanics MM2 force field parameters were used for all calculations. In preliminary work, a graphene bilayer consisting of 127 benzene rings per layer was used to represent the model surface (see Figure 1). Initially, either a benzene or trinitrotoluene (TNT) molecule was placed adjacent to the surface to model adsorption. TNT was chosen as a common explosive molecule for initial consideration since it contains functional similarities with many other explosive molecules [10] [11]. The benzene was modeled on the surface to provide a comparison of binding energy values relative to TNT on the graphene bilayer. The isolated benzene and TNT molecules along with graphene bilayer were optimized based on MM2 parameters. The molecule initially is placed too close to the surface and allowed to be pushed away to the optimal distance. Because of 


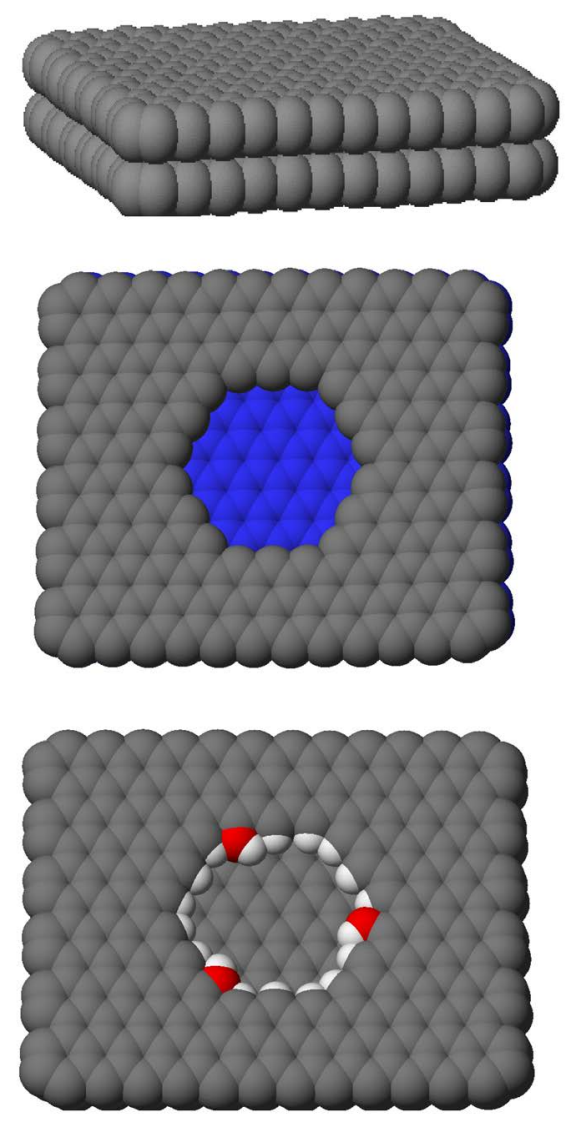

Figure 1. Surface models including: graphene bilayer with two 127-ring layers, twodimensional hexagonal pore with 18 perimeter carbon atoms (bonded hydrogens within pore not shown), and hydroxylated pore containing three $\mathrm{OH}$ groups available for hydrogen bonding interactions with adsorbed molecules.

the uniformity of the surface, there are only small variations in the binding energy, E, based on the exact position relative to the hexagonal arrangement of carbon atoms. The adsorption computations based on Equation (1) produced $\mathrm{E}$ values of $9.4 \mathrm{kcal} / \mathrm{mol}$ for benzene and $17.9 \mathrm{kcal} / \mathrm{mol}$ for TNT on the flat graphene bilayer. The binding energy for TNT was almost entirely due to van der Waal's forces.

At this point, the objective was to enhance the adsorption of TNT on the surface. To do so, a specially designed pore was created within the top layer of graphene to increase molecule-surface interaction. Many prototype pores were considered but the one that displayed the best enhanced adsorption effects is seen in Figure 1. For ease of viewing the pore shape, the hydrogen atoms in the interior of the pore are not shown. The surface pore was hexagonal with three exposed carbons on each edge, which together resulted in 18 perimeter carbons. Each of the perimeter carbons had hydrogen atoms bonded to them, which stayed in the 
$\mathrm{sp}^{2}$ plane of the graphene layer after optimization. This pore increased the TNTE value from 17.9 on the flat bilayer to $24.5 \mathrm{kcal} / \mathrm{mol}$ in the porous bilayer. Again, most of the interaction was from van der Waal's forces, and the newly designed pore successfully attracted the TNT molecule down into the bilayer's pore. In a prior study, the adsorption was enhanced for different shaped polycyclic aromatic hydrocarbons $(\mathrm{PAH})$ by pores with matching shaped flat graphene bilayer pores [28].

Since the TNT was effectively attracted into the pore, the next objective was to further increase the binding energy. Hydroxyl groups were added to the pore's perimeter carbons to increase molecule-surface interaction. Due to widely seen nitro functional group in explosive molecules, adding hydroxyls could potentially raise the interaction for not just TNT but for many explosive molecules via hydrogen bonding. TNT was again used as the initial trial molecule for each prototype pore. In this prototype analysis, six additional models of the pore were created with one to six hydroxyl groups within the inner ring of the pore. The most promising pore was the model with three symmetrically spaced hydroxyl groups (see Figure 1).

This hydroxylated model pore increased the TNT-surface binding energy to $\mathrm{E}=42.3 \mathrm{kcal} / \mathrm{mol}$. This increase was a net increase of $24.4 \mathrm{kcal} / \mathrm{mol}$ relative to the original flat bilayer graphene. The interaction more than doubled from the flat bilayer $\mathrm{E}$ value. The energy from hydrogen bonding increased from 0 on the flat bilayer to $20.9 \mathrm{kcal} / \mathrm{mol}$ in the hydroxylated pore. Enhanced adsorption is due to more favorable combined molecule-surface steric energy. However, values are reported throughout this work as positive desorption values such that larger values mean enhanced molecule-surface site binding energy and thus greater adsorption. Positive values represent the energy required to desorb or remove the molecule from the surface or binding site. The energy contribution from van der Waals interaction increased only slightly by about $2 \mathrm{kcal} / \mathrm{mol}$. Since the hydroxylated pore showed a large increase in molecule-surface interaction with TNT, it was predicted that other explosive molecules with similar functionality would also have enhanced adsorption.

Molecular mechanics force field calculations are based on a sum of covalent and noncovalent energies. Each type of energy has a given functional form and empirical parameters based on atoms involved that match available experimental data. The MM2 covalent bond related energy contributions include stretch, stretch-bend, angle, dihedral, improper torsion. The MM2 noncovalent energy contributions include electrostatics, hydrogen-bonding, and van der Waals. In this study, vdW interactions between the surface carbon atoms and the molecule atoms contribute to the molecule-surface binding energies. Hydrogen bonding between the molecule and surface $\mathrm{OH}$ groups was the other primary source of molecule-surface attraction. The other parameters were relatively unchanged.

Including TNT, a total of 22 explosive molecules were considered [10] [11]. For convenience common abbreviations were used and the molecules examined 
included: TNT, DADNE, CL-20, DNT, HMX, HNS, NTO, PETN, RDX, TATB, TNB, TNR, BDNFA, BTTN, EGDN, K10A, K10B, MTN, NIMMO, NG, TETRYL, and TNAZ. The appropriate names along with these abbreviations are given in Table 1. These molecules come from several of the six broad classes of explosive molecules. The six classes are aliphatic nitro compounds, nitroaromatic compounds, nitramines, nitrate esters, acids salts, and organic peroxides. The 22 molecules are commercially manufactured for various military uses or industrial demolitions [10] [11] [26] [31]. A list of some specific uses for the explosive molecules is shown in Table 2. The 22 molecules expressed functionality similarities including nitro groups, amine groups, aromaticity, and a few with hydroxyl groups.

To investigate the hydrogen bonding energy contributions per hydrogen bond, a comparison with water experimental data was made. From Raman spectroscopy, the energy contribution of hydrogen bonding in a water cluster (30 to 40 molecules in size) have been reported to be about 2.2 to $2.6 \mathrm{kcal} / \mathrm{mol}$ [32]. To find if the hydrogen bond energy contribution from MM2 was within this range, several smaller water clusters were modeled. A pair of water molecules, three

Table 1. Molecule abbreviation and name for 22 explosive compounds.

\begin{tabular}{|c|c|c|}
\hline & Abbreviation & Molecule Name \\
\hline 1 & TNT & 2-methyl-1,3,5-trinitrobenzene \\
\hline 2 & DADNE & 2,2-dinitroethene-1,1-diamine \\
\hline 3 & CL-20 & $2,4,6,8,10,12$-hexanitrohexaazaisowurtzitane \\
\hline 4 & DNT & 1-methyl-2,4-dinitrobenzene \\
\hline 5 & HMX & octahydro-1,3,5,7-tetranitro-1,3,5,7-tetrazocine \\
\hline 6 & HNS & 1,3,5-trinitro-2-[2-(2,4,6-trinitrophenyl)ethenyl]benzene \\
\hline 7 & NTO & 5-nitro-1,2-dihydro-1,2,4-triazol-3-one \\
\hline 8 & PETN & [3-nitrooxy-2,2-bis(nitrooxymethyl)propyl] nitrate \\
\hline 9 & RDX & 1,3,5-trinitro-1,3,5-triazinane \\
\hline 10 & TATB & 2,4,6-trinitrobenzene-1,3,5-triamine \\
\hline 11 & TNB & 1,3,5-trinitrobenzene \\
\hline 12 & TNR & 2,4,6-trinitrobenzene-1,3-diol \\
\hline 13 & BDNFA & bis-dinitropropylformal \\
\hline 14 & BTTN & butane-1,2,4-triol trinitrate \\
\hline 15 & EGDN & ethylene glycol dinitrate \\
\hline 16 & $\mathrm{~K} 10 \mathrm{~A}$ & 2,6-dinitroethylbenzene \\
\hline 17 & K10B & 2,4,6-trinitroethylbenzene \\
\hline 18 & TMETN & [2-methyl-3-nitrooxy-2-(nitrooxymethyl)propyl]nitrate \\
\hline 19 & NIMMO & 3-nitratomethyl-3-methyl oxetane \\
\hline 20 & NG & 1,2,3-trinitroxypropane \\
\hline 21 & Tetryl & 2,4,6-trinitrophenylmethylnitramine \\
\hline 22 & TNAZ & 1,3,3-trinitroazetidine \\
\hline
\end{tabular}


Table 2. Common uses of the 22 explosive compounds modelled in this work.

\begin{tabular}{|c|c|c|}
\hline & Molecule Abbreviation & Common Use of Molecule \\
\hline 1 & TNT & Component of dynamite \\
\hline 2 & DADNE & Detonation and gun propellant \\
\hline 3 & CL-20 & Rocket propellant \\
\hline 4 & DNT & Precursor of TNT, plasticizer for explosives \\
\hline 5 & HMX & Polymer bonded-explosives for nuclear detonation \\
\hline 6 & HNS & Mortar grenades \\
\hline 7 & NTO & Pressed thermoplastic explosives \\
\hline 8 & PETN & Plastic explosive \\
\hline 9 & RDX & Plastic explosive mix \\
\hline 10 & TATB & Nuclear warhead charge/explosive charges \\
\hline 11 & TNB & Commercial mining explosive \\
\hline 12 & TNR & Lead salts/ignition agent \\
\hline 13 & BDNFA & Energetic plasticizer \\
\hline 14 & BTTN & Propellant \\
\hline 15 & EGDN & Precursor \\
\hline 16 & $\mathrm{~K} 10 \mathrm{~A}$ & Polymer bonded explosives \\
\hline 17 & $\mathrm{~K} 10 \mathrm{~B}$ & Polymer bonded explosives \\
\hline 18 & MTN & Plasticizer and propellant \\
\hline 19 & NIMMO & Plasticizer \\
\hline 20 & NG & High explosive \\
\hline 21 & Tetryl & Base charge of blasting caps \\
\hline 22 & TNAZ & Solid rocket and gun propellant \\
\hline
\end{tabular}

water molecules, four water molecules, and seven water molecules were modeled. Each cluster of molecules was optimized and the stabilization energy due to hydrogen bonding relative to the same number of isolated molecules was found. The total hydrogen bonding energy for the cluster was divided by the number of bonds in the cluster to generate the energy per hydrogen bond. The interaction energy per hydrogen bond was found to be $2.5,2.6,2.3$, and $2.6 \mathrm{kcal} / \mathrm{mol}$ for two, three, four, and seven water molecules, respectively. These calculated values were within the range of reported experimental values so we accept that MM2 calculations can provide reasonable estimates of hydrogen bonding contributions.

Each explosive molecule of interest (Table 1) was placed on the flat bilayerand in the hydroxylated pore site (see Figure 1) and optimized by MM2 to maximize interactions and to achieve its lowest energy. This rearrangement, for the minimization of the geometry, took place in the hydroxylated pore. After initial placement, molecule movement often repositioned the molecule nitro groups optimally toward some of the hydroxyl groups. The interaction energy was determined from Equation (1) for each of the 22 explosive molecules. 
The molecular mechanics using MM2 parameters was able to calculate hydrogen bonding's contributing energy for the explosive molecules in the hydroxylated pores. The stabilization energy for each of the 22 molecules was found. Based on acceptable hydrogen bond distances, the number of hydrogen bonds between the molecule and surface pore was determined. The average hydrogen bond energies found was compared to the accepted range. The average organic molecular hydrogen bond was reported to be approximately 3.0 to 5.0 $\mathrm{kcal} / \mathrm{mol}[33]$.

\section{Results and Discussion}

To summarize the trend noted for TNT-surface interactions E $(\mathrm{kcal} / \mathrm{mol})$ were 17.9 for the graphene bilayer, 24.5 for the surface layer hexagonal pore, and 42.3 for the hydroxylated hexagonal pore. As shown below, similar increases were observed for the other explosive molecules examined in this work.

Table 3 shows the interaction energies, E, for the 22 molecules with the flat

Table 3. Comparison of molecule-surface binding energies for the flat graphene bilayer surface and the hydroxylated pore surface along with the enhancement due to pore adsorption.

\begin{tabular}{|c|c|c|c|}
\hline Molecule & $\begin{array}{c}\mathrm{E} \\
\text { (flat bilayer) } \\
\mathrm{kcal} / \mathrm{mol}\end{array}$ & $\begin{array}{c}\mathrm{E} \\
\text { (hydroxy pore) } \\
\mathrm{kcal} / \mathrm{mol}\end{array}$ & $\begin{array}{c}\mathrm{E} \\
\text { (enhancement) } \\
\mathrm{kcal} / \mathrm{mol}\end{array}$ \\
\hline TNT & 17.9 & 42.3 & 24.4 \\
\hline DADNE & 14.4 & 22.5 & 8.1 \\
\hline CL-20 & 14.9 & 38.3 & 23.4 \\
\hline DNT & 15.5 & 35.3 & 19.8 \\
\hline HMX & 15.1 & 37.3 & 22.2 \\
\hline HNS & 24.7 & 32.3 & 7.6 \\
\hline NTO & 11.2 & 20.5 & 9.3 \\
\hline PETN & 14.7 & 35.2 & 20.5 \\
\hline RDX & 7.2 & 21.5 & 14.3 \\
\hline TATB & 24.5 & 51.5 & 27.0 \\
\hline TNB & 23.7 & 53.5 & 29.8 \\
\hline TNR & 19.2 & 44.3 & 25.1 \\
\hline BDNFA & 15.9 & 33.4 & 17.5 \\
\hline BTTN & 14.2 & 27.0 & 12.8 \\
\hline EGDN & 10.8 & 20.7 & 9.9 \\
\hline $\mathrm{K} 10 \mathrm{~A}$ & 17.3 & 27.2 & 9.9 \\
\hline $\mathrm{K} 10 \mathrm{~B}$ & 19.5 & 48.5 & 29.0 \\
\hline MTN & 12.7 & 34.6 & 21.9 \\
\hline NIMMO & 10.2 & 24.4 & 14.2 \\
\hline NG & 11.8 & 37.3 & 25.5 \\
\hline Tetryl & 15.5 & 32.7 & 17.2 \\
\hline TNAZ & 17.2 & 23.6 & 6.4 \\
\hline
\end{tabular}


graphitic bilayer and with the hydroxylated pore. Each of the 22 adsorbate molecules showed increased molecule-surface interaction on the bilayer hydroxylated pore as compared to the flat bilayer. A significant adsorption binding energy enhancement was found for all these explosives compounds just as it was for TNT (see Table 3). The average $\mathrm{E}$ for all the molecules with the flat graphite surface was $15.8 \mathrm{kcal} / \mathrm{mol}$. The average $\mathrm{E}$ for all the molecules for the hydroxy pore was $33.8 \mathrm{kcal} / \mathrm{mol}$. The increase in adsorption energy was determined from $\mathrm{E}$ (enhancement $)=\mathrm{E}$ (hydroxy pore $)-\mathrm{E}$ (flat bilayer) . The average enhancement of the molecule-surface binding energy was $18.0 \mathrm{kcal} / \mathrm{mol}$ which is more than a doubling of the Erelative to the flat surface.

Some of the larger molecules such as HNS, PETN, C1-20, BNDFA, and BTTN would only fit partially into the pore but were still able to form multiple new hydrogen bonds. Some of the molecules had internal hydrogen bonds but these did not contribute to the stabilization binding energy within the pore since they were already present prior to adsorption.

The formation of hydrogen bonds between the molecule and pore is illustrated in Figure 2 that shows TNT within the hydroxy pore and the H-bonds marked by internuclei separation. Note that the hydrogen bonds could be with one $\mathrm{OH}$ hydrogen shared with two oxygens as in TNT or could be between an $\mathrm{OH}$ hydrogen and a single oxygen. In addition, some of the molecules had internal hydrogen bonds that remained unchanged and did not contribute to new molecule-surface interaction. For example, TATB has 6 intramolecular hydrogen bonds and 6 new molecule-surface pore bonds (see Figure 2). Only the latter are counted in consideration of a hydrogen bond analysis.

The 22 explosive molecules were found to have a total of 96 hydrogen bonds between the molecule and pore. This number (shown in Table 4) is the average stabilization energy from all the hydrogen bonds formed between the molecule and hydroxylated pore. The contributions of the hydrogen bonding to the molecule-surface interaction are shown in Table 4 along with the number of hydrogen bonds and the average energy per bond. Note that the average of the 96 hydrogen bonds gave an energy of $4.2 \mathrm{kcal} / \mathrm{mol}$ and a standard deviation of 0.7 $\mathrm{kcal} / \mathrm{mol}$. As noted previously the internal hydrogen bonds within any of the molecules did not contribute to this count. This average agrees well with the reported organic molecular hydrogen bond energy of 3.0 to $5.0 \mathrm{kcal} / \mathrm{mol}$ [33].

The hydrogen bond contribution to $\mathrm{E}$ (see Table 4) may be given as a percent of the of total molecule-surface interaction energy $\mathrm{E}$ (see Table 3). For the 22 molecules, the percent of $\mathrm{E}$ due to $\mathrm{H}$-bond formation varied from a low of $36.7 \%$ to a high of $72.5 \%$ with an average of $53.2 \%$. The average hydrogen bond length as about $0.22 \mathrm{~nm}$.

The van der Waals forces calculated for the graphene bilayer hydroxylated pore were increased only slightly for each molecule. The E vdW going form the flat bilayer to the hydroxylated pore increased the interaction by only a few $\mathrm{kcal} / \mathrm{mol}$ on average. The initial and continuing vdW contribution remained about the same while the movement of the molecule in the pore added new and 


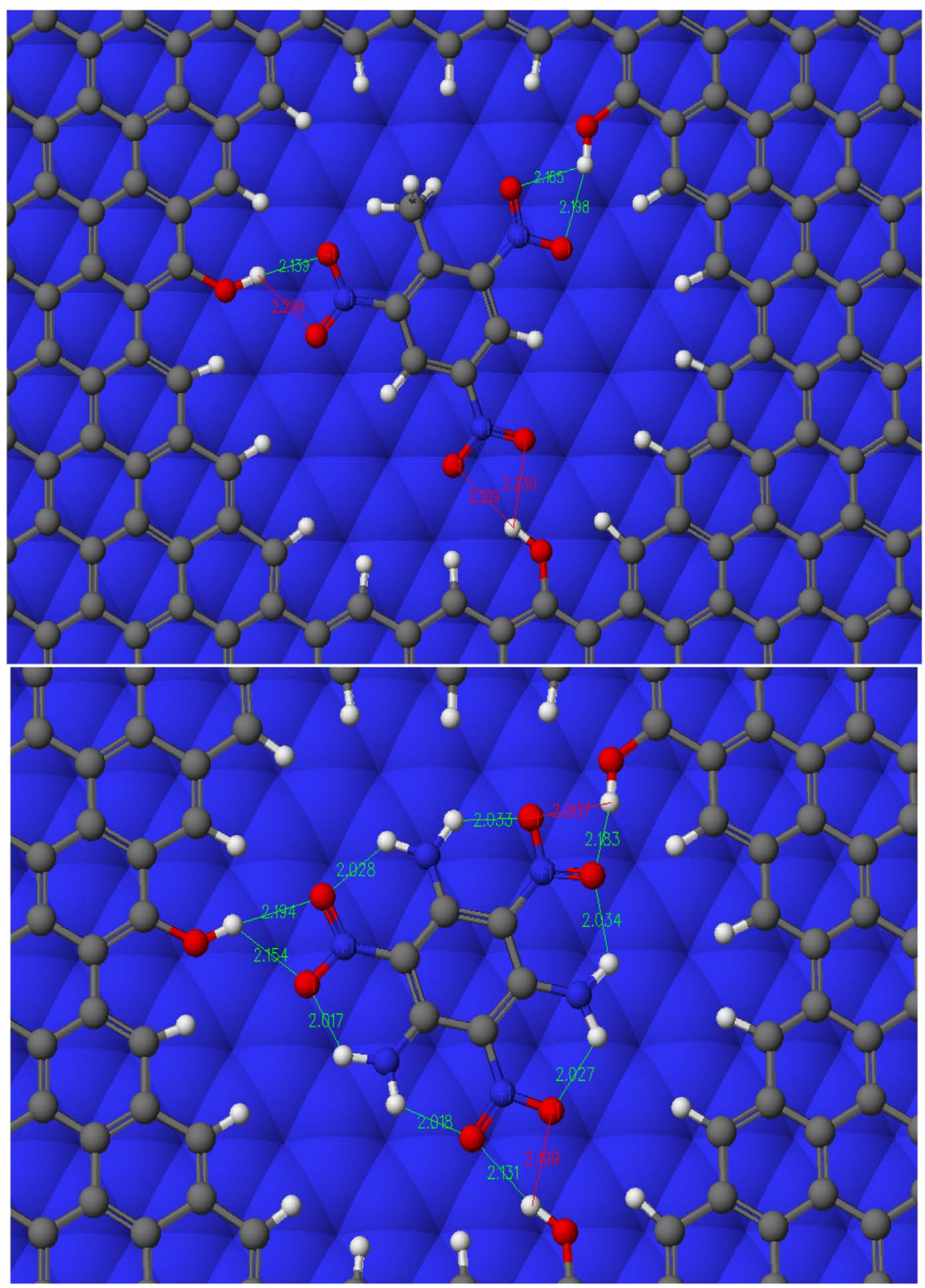

Figure 2. TNT in hydroxylated pore (top) with 6 molecule-surface hydrogen bonds formed and TATB in hydroxylated pore (bottom) with 6 internal hydrogen bonds and 6 molecule-surface hydrogen bonds.

significant hydrogen bonding.

Based on the results from the computational analysis of 22 explosive molecules on a nonporous graphene bilayer and a hydroxylated porous graphene bilayer, it was observed that a substantial interaction exists between the molecules and hydroxylated pore model. The adsorption was calculated from molecule-surface binding energy. The adsorption increased considerably due to the presence of hydrogen bonding. Since the bilayer model already had a significant contribution of van der Waals forces attracting into the surface, the hydroxylated pore proved to effectively enhance the total binding energy in the form of 
Table 4. Molecule-surface binding energydue to hydrogen bonding, E(H-bond), for the hydroxylated pore, the number of hydrogen bonds formed with the pore, and the average hydrogen bond strength due to the molecule pore interaction.

\begin{tabular}{|c|c|c|c|}
\hline Molecule & $\begin{array}{l}\text { E(H-bond) } \\
\mathrm{kcal} / \mathrm{mol}\end{array}$ & H-bonds & $\begin{array}{c}\text { H-bond strength } \\
\mathrm{kcal} / \mathrm{mol}\end{array}$ \\
\hline TNT & 20.9 & 6 & 3.5 \\
\hline DADNE & 11.7 & 2 & 5.9 \\
\hline CL-20 & 19.2 & 4 & 4.8 \\
\hline DNT & 14.7 & 4 & 3.7 \\
\hline HMX & 17.2 & 4 & 4.3 \\
\hline HNS & 14.7 & 3 & 4.9 \\
\hline NTO & 10.1 & 2 & 5.1 \\
\hline PETN & 22.1 & 5 & 4.4 \\
\hline RDX & 8.0 & 2 & 4.0 \\
\hline TATB & 32.5 & 6 & 5.4 \\
\hline TNB & 22.6 & 6 & 3.8 \\
\hline TNR & 24.5 & 6 & 4.1 \\
\hline BDNFA & 18.7 & 4 & 4.7 \\
\hline BTTN & 17.9 & 4 & 4.5 \\
\hline EGDN & 15.0 & 4 & 3.8 \\
\hline $\mathrm{K} 10 \mathrm{~A}$ & 14.3 & 4 & 3.6 \\
\hline K10B & 17.8 & 6 & 3.0 \\
\hline MTN & 19.2 & 5 & 3.8 \\
\hline NIMMO & 10.9 & 3 & 3.6 \\
\hline NG & 22.6 & 6 & 3.8 \\
\hline Tetryl & 20.6 & 5 & 4.1 \\
\hline TNAZ & 15.9 & 5 & 3.2 \\
\hline
\end{tabular}

hydrogen bonding. All of the 22 explosive molecules exhibited an increased attraction to the hydroxylated porous surface. A nanosensor based on hydroxylate pores could enhance the surface affinity for various explosive molecules.

An effective sensor for a very low vapor pressure explosive molecule would have to overcome interference from other atmospheric molecules like $\mathrm{N}_{2}, \mathrm{O}_{2}, \mathrm{Ar}$, $\mathrm{CO}_{2}$, and $\mathrm{H}_{2} \mathrm{O}$ or even smaller amounts of substances like $\mathrm{CH}_{4}, \mathrm{NO}_{2}, \mathrm{~N}_{2} \mathrm{O}$, and small hydrocarbons present in trace amounts in the atmosphere that could interact with thesensor surface. Some common and less common atmospheric gas molecules like $\mathrm{N}_{2}, \mathrm{O}_{2}, \mathrm{CO}_{2}, \mathrm{H}_{2} \mathrm{O}, \mathrm{CH}_{4}, \mathrm{NO}_{2}$, and $\mathrm{N}_{2} \mathrm{O}$ were modeled on the hydroxylated poreand tended to have $\mathrm{E}$ values in the 2 to $12 \mathrm{kcal} / \mathrm{mol} \mathrm{range.}$

Due to the exponential portion in Equation (2), one can understand that the larger the binding affinity of TNT or other explosive molecules, the more its adsorption could still be significant even in trace amounts. To explore the ratio of explosive molecules adsorbing on the hydroxylated pore, a ratio of adsorption calculation was conducted to analyze this property. Using Equation (2), the ad- 
sorption behavior of two different molecules on the same surface or the same molecule on two different surfaces can be compared. This equation is based on low concentration so while appropriate for a very low concentration, it is less appropriate for higher concentration species. However the details may vary, there remains a ratio that is affected by the differences of the binding energies $\mathrm{E}$. To explore the effect of this ratio, we note that the exponential portion of the equation can greatly favor the higher E molecule.

In prior studies molecular mechanics calculated binding energies have been shown to provide good representations of experimental binding energies provided that the surface is modeled appropriately [24]. The term $\exp (\mathrm{E} / \mathrm{RT})$ or $\mathrm{e}^{\mathrm{E} / \mathrm{RT}}$ where $\mathrm{E}$ is written as a positive value will dominate as $\mathrm{E}$ increases. Hence a surface that can enhance E will greatly increase the amount of adsorption and thus the sensitivity of a nanosensor. Consider an example where $\mathrm{E}$ would be the calculated binding energy for TNT vapor and another E is the binding energy for some naturally occurring atmospheric molecules. Although an explosive such as TNT might be present in very trace amounts, if the surface interaction energy is great enough then because of the exponential factor in Equation (2), the equilibrium adsorption may be sufficient to still favor adsorption of TNT vapor or some other explosive molecule.

To illustrate the effect of this ratio in Equation (2), consider one molecule that has a value of $\mathrm{E} 1=10.0 \mathrm{kcal} / \mathrm{mol}$ and the other molecule to have $\mathrm{E} 2$ values of $15.0,20.0,25.0,30.0$, or $35.0 \mathrm{kcal} / \mathrm{mol}$. At $298 \mathrm{~K}$ these energies give rise to $\mathrm{e}^{(\mathrm{E2} 2 \mathrm{E} 1) / \mathrm{RT}}$ values of $4.7 \times 10^{3}, 2.2 \times 10^{7}, 1.0 \times 10^{11}$, and $4.7 \times 10^{14}, 2.2 \times 10^{18}$, respectively. For this range of values, the square root of the ratio $E_{2} / E_{1}$ varies only by $0.82,0.71,0.63,0.58$, and 0.53 , respectively. In other words, the effect of the exponential term dominates and could offset quite significant differences in partial pressure that could give a very low ratio for partial pressure ratios, $\mathrm{P}_{1} / \mathrm{P}_{2}$.

One other illustration of the exponential factor is to consider the adsorption of TNT that increased from $\mathrm{E}$ (flat) $=17.9 \mathrm{kcal} / \mathrm{mol}$ on the flat surface to $\mathrm{E}(\mathrm{OH}$ pore $)=42.3 \mathrm{kcal} / \mathrm{mol}$ on the hydroxylated pore. This increase in $\mathrm{E}$ corresponds to $\exp [\mathrm{E}(\mathrm{OH}$ pore $)-\mathrm{E}(\mathrm{flat})] / \mathrm{RT}$ or a value of about $7.7 \times 10^{17}$. The enhanced $\mathrm{E}$ creates a more sensitive nanosensor.

Molecular dynamics could be useful to show how the TNT molecule would interact while surrounded by other molecules. The affinity for TNT or other explosive molecules must be sufficiently greater than the molecules naturally occurring in the atmosphere and must be able to modify the surface enough through adsorption to achieve a detectable signal. The positions of each explosive molecule's nitro group or groups were done to maximize the interaction of hydrogen bonding. The range of all variations of placement was not considered but only a representative placement. Additional investigation of placement variation would be useful.

\section{Conclusions}

Force field calculations provide a computationally rapid means to deal with sys- 
tems incorporating a large number of atoms. These classical molecular mechanics calculations can provide useful estimates of molecule-surface interactions. In this work, the enhancement of molecule-surface interaction was provided by the construction of a model graphene bilayer hydroxylated pore. In comparison to an unmodified graphene bilayer, there was a significant increase in the surface binding energy. Some of this increase was due to slightly enhanced vdW forces as the molecules resided within the shallow two-dimensional pore. However, most of the enhanced molecule-surface interaction was due to a significant increase in hydrogen bonding.

For example, in the specific case of 2-methyl-1,3,5-trinitrobenzene, the TNTsurface interactions were $17.9 \mathrm{kcal} / \mathrm{mol}$ for the flat graphene bilayer and 42.3 $\mathrm{kcal} / \mathrm{mol}$ for the hexagonal hydroxylated pore. Similarly, all 22 explosive molecules examined in this work displayed increased adsorption in the hydroxylated pore. The initial vdW interaction with the surface remained fairly constant while the overall interaction was increased due to hydrogen bonding with the hydroxyl groups of the pore (see Table 3).

Because of the similarity of a wide variety of explosive molecules with regard to being hydrogen bonding acceptors, the three hydrogen bonding donor $\mathrm{OH}$ groups in the pores were effective in forming one to multiple molecule-surface hydrogen bonds. The average increase in binding energy going from the flat graphene bilayer to the three $\mathrm{OH}$ group hydroxylated pore for the 22 molecules examined was $17.0 \mathrm{kcal} / \mathrm{mol}(0.737 \mathrm{eV}$ or $71.1 \mathrm{~kJ} / \mathrm{mol})$. About $94 \%$ of this increased molecule-surface interaction was due to additional molecule-surface hydrogen bonding. The average ratio of the interaction energies was $\mathrm{E}(\mathrm{OH}$ pore $) /$ $\mathrm{E}$ (bilayer) $=2.20$ with a standard deviation of 0.54 . Thus, the presence of the hydroxylated pore, on average, more than doubled the surface interaction relative to the regular graphene surface for these common explosive molecules.

This study suggests that there may be potential for using a graphene bilayer with a hydroxylated pore in the detection of explosive molecules. Such a hydroxylated pore could, in theory, be used as a nanosensor to detect trace amounts of explosive molecules in the air. Because of the large attraction, the hydroxylated pore has for explosive molecules, it might provide the high sensitivity needed for detecting trace amounts of explosives. Graphene bilayers would have to have multiple hydroxylated pores constructed or possibly surface modification by adsorption of suitable hydrogen donor molecules. The modified hydroxylated graphene surface could then be incorporated into a device that responded to adsorption of explosive molecules into the pores or on the surface by a measurable change in some property such as electrical resistance.

The present computational analysis suggests that further research related to the adsorption of explosive molecules on graphene or modified graphene surfaces would be useful. Other hydroxylated pores or surfaces should be considered in the future to estimate the binding energies associated with explosive molecules. Exploring placement of molecules on a surface or in a pore would be useful, and exploring the model outside of in vacuo conditions and considering 
the effects of humidity would be essential.

\section{Conflicts of Interest}

The authors declare no conflicts of interest regarding the publication of this paper.

\section{References}

[1] Zhang, J., Song, L., Zhang, Z.P., Chen, N. and Qu, L.T. (2014) Environmentally Responsive Graphene Systems. Small, 10, 2151-2164.

https://doi.org/10.1002/smll.201303080

[2] Kwon, O.S., Park, S.J., Jang, J. and Bae, J. (2016) Novel Graphene Sensors for Chemical and Biological Applications. In: Aliofkhazraei, M., Ed., Graphene Science Handbook: Fabrication Methods, CRC Press, Boca Raton, FL, 269-286.

[3] Yavari, F. and Koratkar, N. (2012) Graphene-Based Chemical Sensors. Journal of Physical Chemistry Letters, 3, 1746-1753. https://doi.org/10.1021/jz300358t

[4] Wisitsoraat, A. and Tuantranont, A. (2013) Graphene-Based Chemical and Biosensors. In: Tuantranont, A., Ed., Applications of Nanomaterials in Sensors and Diagnostics. Springer Series on Chemical Sensors and Biosensors (Methods and Applications), Springer, Berlin, Heidelberg, 103-141.

[5] Chung, M.G., Kim, D.H., Lee, H.M., Kim, T., Choi, J.H., Seo, D.K., Yoo, J.-B., Hong, S.-H., Kang, T.J. and Kim, Y.H. (2012) Highly Sensitive $\mathrm{NO}_{2}$ Gas Sensor Based on Ozone Treated Graphene. Sensors and Actuators, B: Chemical, 166-167, 172-176.

[6] Moore, D.S. (2004) Instrumentation for Trace Detection of High Explosives. Review of Scientific Instruments, 75, 2499-2512. https://doi.org/10.1063/1.1771493

[7] Moore, D.S. (2007) Recent Advances in Trace Explosives Detection Instrumentation. Sensing and Imaging. An International Journal, 8, 9-38. https://doi.org/10.1007/s11220-007-0029-8

[8] Yi, D., Larry Senesac, L.R. and Thundat, T.G. (2008) Speciation of Energetic Materials on a Microcantilever Using Surface Reduction. Scanning, 30, 208-212. https://doi.org/10.1002/sca.20096

[9] Zhang, J. and Fahrenthold, E.P. (2019) Graphene-Based Sensing of Gas-Phase Explosives. ACS Applied Nano Materials, 2, 1445-1456.

[10] Akhavan, J. (2011) The Chemistry of Explosives. 3rd Edition, RSC Publishing, Cambridge.

[11] Klapotke, T.M. (2011) Chemistry of High-Energy Materials. De Gruyter Publishers, Berlin, Germany. https://doi.org/10.1515/9783110227840

[12] Rahim, I., Shah, M., Iqbal, M. and Khan, A. (2018) Synthesis, Structural, Optical, Morphological and Multi Sensing Properties of Graphene Based Thin Film Devices. Materials Research Express, 5, Article ID: 096403. https://doi.org/10.1088/2053-1591/aac98b

[13] Ye, H., Nallon, E.C., Schnee, V.P., Shi, C., Jiang, K., Xu, J., Feng, S., Wang, H. and Li, Q. (2018) Enhance the Discrimination Precision of Graphene Gas Sensors with a Hidden Markov Model. Analytical Chemistry, 90, 13790-13795. https://doi.org/10.1021/acs.analchem.8b04386

[14] Lee, J., Lee, C.-J., Kang, J., Park, H., Kim, J., Choi, M. and Park, H. (2019) Multifunctional Graphene Sensor for Detection of Environment Signals Using a De- 
coupling Technique. Solid-State Electronics, 151, 40-46. https://doi.org/10.1016/j.sse.2018.10.014

[15] Nallon, E.C., Schnee, V.P., Bright, C., Polcha, M.P. and Li, Q.L. (2016) Chemical Discrimination with an Unmodified Graphene Chemical Sensor. ACS Sensors, 1, 26-31. https://doi.org/10.1021/acssensors.5b00029

[16] Melios, C., Panchal, V., Edmonds, K., Lartsev, A., Yakimova, R. and Kazakova, O. (2018) Detection of Ultralow Concentration $\mathrm{NO}_{2}$ in Complex Environment Using Epitaxial Graphene Sensors. ACS Sensors, 3, 1666-1674.

https://doi.org/10.1021/acssensors.8b00364

[17] Hannon, A., Lu, Y., Li, J. and Meyyappan, M. (2016) A Sensor Array for The Detection and Discrimination of Methane and Other Environmental Pollutant Gases. Sensors, 16, 1163. https://doi.org/10.3390/s16081163

[18] Rattanabut, C., Wongwiriyapan, W., Muangrat, W., Bunjongpru, W., Phonyiem, M. and Song, Y.J. (2018) Graphene and Poly(Methyl Methacrylate) Composite Laminates on Flexible Substrates for Volatile Organic Compound Detection. Japanese Journal of Applied Physics, 57, 04FP10. https://doi.org/10.7567/JJAP.57.04FP10

[19] Park, S., Park, M., Kim, S., Yi, S.-G., Kim, M., Son, J., Cha, J., Hong, J. and Yoo, K.-H. (2017) $\mathrm{NO}_{2}$ Gas Sensor Based on Hydrogenated Graphene. Applied Physics Letters, 111, Article ID: 213102. https://doi.org/10.1063/1.4999263

[20] Leenaerts, O., Partoens, B. and Peeters, F.M. (2007) Adsorption of $\mathrm{H}_{2} \mathrm{O}, \mathrm{NH}_{3}, \mathrm{CO}$, $\mathrm{NO}_{2}$, and $\mathrm{NO}$ on Graphene: A First-Principles Study. Physical Review B, 77, 125416. https://doi.org/10.1103/PhysRevB.77.125416

[21] Peng, Y. and Li, J. (2013) Ammonia Adsorption on Graphene and Graphene Oxide: A First-Principles Study. Frontiers of Environmental Science \& Engineering, 7, 403-411. https://doi.org/10.1007/s11783-013-0491-6

[22] Rouhani, M. (2019) DFT Study on Adsorbing and Detecting Possibility of Cyanogen Chloride by Pristine, B, Al, Ga, Si And Ge Doped Graphene. Journal of Molecular Structure, 1181, 518-535. https://doi.org/10.1016/j.molstruc.2019.01.006

[23] Zheng, Z. and Wang, H. (2019) Different Elements Doped Graphene Sensor for $\mathrm{CO}_{2}$ Greenhouse Gases Detection: The DFT Study. Chemical Physics Letters, 721, 33-37. https://doi.org/10.1016/j.cplett.2019.02.024

[24] Son, J.H. and Rybolt, T.R. (2013) Force Field Based MM2 Molecule-Surface Binding Energies for Graphite and Graphene. Graphene, 2, 18-34. https://doi.org/10.4236/graphene.2013.21004

[25] Agrawal, J.P. and Hodgson, R. (2007) Organic Chemistry of Explosion. Wiley, New York. https://onlinelibrary.wiley.com/doi/book/10.1002/9780470059364 https://doi.org/10.1002/9780470059364

[26] Yinon, J. (2002) Field Detection and Monitoring of Explosives. Trends in Analytical Chemistry, 21, 292-301. https://doi.org/10.1016/S0165-9936(02)00408-9

[27] Rybolt, T.R., Wells, C.E., Sisson, C.R., Black, C.B. and Ziegler, K.A. (2007) Evaluation of Molecular Mechanics Calculated Binding Energies for Isolated and Monolayer Organic Molecules on Graphite. Journal of Colloid and Interface Science, 314, 434-445. https://doi.org/10.1016/j.jcis.2007.05.083

[28] Rybolt, T.R. and Black, C.B. (2017) Polycyclic Aromatic Hydrocarbon MoleculeSurface Binding Energies in Site Specific Graphene Bilayer Nanopores: A Puzzle-ene Force Field Calculation. Graphene, 6, 72-84. https://doi.org/10.4236/graphene.2017.63006

[29] Frye, C.W. and Rybolt, T.R. (2018) Nanohashtag Structures Based on Carbon Na- 
notubes and Molecular Linkers. Surface Science, 669, 34-44.

https://doi.org/10.1016/j.susc.2017.11.005

[30] Rybolt, T.R., Logan, D.L., Milburn, M.W., Thomas, H.E. and Waters, A.B. (1999) Correlations of Henry's Law Gas-Solid Virial Coefficients and Chromatographic Retention Times for Hydrocarbons and Halocarbons Adsorbed on Carbopack C Carbon. Journal of Colloid and Interface Science, 220, 148-156. https://doi.org/10.1006/jcis.1999.6522

[31] Yinon, J. and Zitrin, S. (1993) Modern Methods and Applications in Analysis of Explosives. John Wiley \& Sons, Chichester, England.

[32] Xantheas, S.S. (2000) Cooperativity and Hydrogen Bonding Network in Water Clusters. Chemical Physics, 258, 225-231. https://doi.org/10.1016/S0301-0104(00)00189-0

[33] Jeffery, G.A. (1997) An Introduction to Hydrogen Bonding. Oxford University Press, Oxford. 